University of Wollongong

Research Online

Australian Institute for Innovative Materials -

Papers

Australian Institute for Innovative Materials

$1-1-2019$

Carbon Nanotube Yarn for Fiber-Shaped Electrical Sensors, Actuators, and Energy Storage for Smart Systems

Yongwoo Jang

Hanyang University

Sung Min Kim

Hanyang University

Geoffrey M. Spinks

University of Wollongong, gspinks@uow.edu.au

Seon Jeong Kim

sjk@hanyang.ac.kr

Follow this and additional works at: https://ro.uow.edu.au/aiimpapers

Part of the Engineering Commons, and the Physical Sciences and Mathematics Commons

Research Online is the open access institutional repository for the University of Wollongong. For further information contact the UOW Library: research-pubs@uow.edu.au 


\title{
Carbon Nanotube Yarn for Fiber-Shaped Electrical Sensors, Actuators, and Energy Storage for Smart Systems
}

\author{
Abstract \\ Smart systems are those that display autonomous or collaborative functionalities, and include the ability \\ to sense multiple inputs, to respond with appropriate operations, and to control a given situation. In \\ certain circumstances, it is also of great interest to retain flexible, stretchable, portable, wearable, and/or \\ implantable attributes in smart electronic systems. Among the promising candidate smart materials, \\ carbon nanotubes (CNTs) exhibit excellent electrical and mechanical properties, and structurally \\ fabricated CNT-based fibers and yarns with coil and twist further introduce flexible and stretchable \\ properties. A number of notable studies have demonstrated various functions of CNT yarns, including \\ sensors, actuators, and energy storage. In particular, CNT yarns can operate as flexible electronic sensors \\ and electrodes to monitor strain, temperature, ionic concentration, and the concentration of target \\ biomolecules. Moreover, a twisted CNT yarn enables strong torsional actuation, and coiled CNT yarns \\ generate large tensile strokes as an artificial muscle. Furthermore, the reversible actuation of CNT yarns \\ can be used as an energy harvester and, when combined with a CNT supercapacitor, has promoted the \\ next-generation of energy storage systems. Here, progressive advances of CNT yarns in electrical \\ sensing, actuation, and energy storage are reported, and the future challenges in smart electronic \\ systems considered.

\section{Disciplines} \\ Engineering | Physical Sciences and Mathematics

\section{Publication Details} \\ Jang, Y., Kim, S. Min., Spinks, G. M. \& Kim, S. Jeong. (2019). Carbon Nanotube Yarn for Fiber-Shaped \\ Electrical Sensors, Actuators, and Energy Storage for Smart Systems. Advanced Materials, Online First \\ $1902670-1-1902670-14$.
}




\section{WILEY-VCH}

Article type: Progress Report

\section{Carbon nanotube yarn as fiber-shaped electrical sensor, actuator, and energy storage for smart system}

Yongwoo Jang ${ }^{l,+}$, Sung Min Kim ${ }^{2,3 \dagger}$, Geoffrey M. Spinks ${ }^{4}$, and Seon Jeong Kim ${ }^{1, *}$

${ }^{1}$ Center for Self-Powered Actuation, Department of Biomedical Engineering, Hanyang University, Seoul 04763, South Korea

${ }^{2}$ Department of Physical Education, Hanyang University, Seoul 04763, South Korea

${ }^{3}$ Department of Active Aging Industry, Hanyang University, Seoul 04763, South Korea

${ }^{4}$ Australian Institute for Innovative Materials, ARC Centre of Excellence for Electromaterials Science, University of Wollongong, Wollongong, NSW 2522, Australia

Keywords: carbon nanotube yarn, smart electronic system, sensor, actuator, energy storage

${ }^{\dagger}$ The authors contributed equally to this work.

*To whom correspondence should be addressed: sjk@hanyang.ac.kr

\section{Address for correspondence:}

Seon Jeong Kim

Director of National Creative Research Initiative Center for Self-Powered Actuation

Professor of Department of Biomedical Engineering, Hanyang University, Seoul 04763, South Korea

Email: sjk@ hanyang.ac.kr

Tel.: +82-2-2220-2321 


\section{WILEY-VCH}

\section{Abstract}

Smart systems are those that display autonomous or collaborative functionalities, and include the ability to sense multiple inputs, to respond with appropriate operations, and to control a given situation. In certain circumstances, it is also of great interest to retain flexible, stretchable, portable, wearable, and/or implantable attributes in a smart electronic system. Among the promising candidate smart materials, carbon nanotube (CNT) exhibits excellent electrical and mechanical properties, and structurally fabricated CNT based fibers and yarns with coil and twist further introduce flexible and stretchable properties. A number of notable studies have demonstrated various functions of CNT yarns, including sensor, actuator, and energy storage. In particular, CNT yarns can operate as flexible electronic sensors and electrodes to monitor strain, temperature, ionic concentration, and concentraion of target biomolecules. Moreover, a twisted CNT yarn enables strong torsional actuation and coiled CNT yarns generate large tensile strokes as an artificial muscle. Furthermore, the reverse actuation function of CNT yarns can be used as an energy harvester and when combined with a CNT supercapacitor has promoted the next-generation energy storage system. Here we report progressive advances of CNT yarns in electrical sensing, actuation, and energy storage, and further consider the future challenges in smart electronic systems. 


\section{WILEY-VCH}

\section{Introduction}

"Smart systems" refer generically to various advanced technologies that are regarded as platforms for next-generation devices in a variety of fields. ${ }^{[1]}$ The principal functions of a smart system are realized by the seamless integration of monitoring and responding systems that react autonomously and appropriately to specific signals. ${ }^{[2]}$ Notable recent advances in smart materials are expected to make it possible for the miniaturization and integration of smart systems into portable wearable or implantable devices for applications including personal health management and for human-machine interactions. ${ }^{[1,3]}$ In general, smart materials are those that display multiple functionalities, including stimuli-responsiveness; the ability to generate, transport and/or store charge; and, in some cases biocompatibility or biodegradability. ${ }^{[4]}$ The integration of smart materials have the potential to revolutionize conventional electronic systems in healthcare, manufacturing, and robotics. ${ }^{[3 \mathrm{a}, 3 \mathrm{~b}]}$

Fiber based smart materials are particularly attractive for many applications. ${ }^{[4]}$ Fiber processing enables the enhancement of mechanical and electrical properties as well as providing control of composition, surface area and porosity. Textile fabrication also allows fibers to be integrated into fabrics with complex architectures. Next generation wearable technologies will be built from textiles incorporating sensor fibers; actuating fibers; and fibers that harness movement or latent heat to generate their own power. ${ }^{[5]}$ Fundamentally important in all of these applications are the need for fibers with high electrical conductivity and mechanical robustness. The sensor and actuator elements are electrically networked for monitoring and control and must be integrated with a suitable power supply. The desire to implement these systems in wearable or implantable applications also requires flexibility, stretchability and resistance to mechanical damage. In line with these needs, carbon nanotube yarns have emerged as ideal candidate materials that demonstrate capabilities as electronic sensor, actuator, and energy harvester. Significant advances in recent times to improve the 


\section{WILEY-VCH}

basic electrical and mechanical properties of these materials have enabled their further development as smart materials. Here we review progress in this field, consider various potential applications for carbon nanotube yarns and summarize the areas for future work.

\section{Properties of Carbon Nanotube Yarns}

Carbon nanotubes (CNTs) are currently the most promising material for fiber based smart systems. The high strength and electrical conductivity of CNT yarns created much initial interest in using these materials for electronic applications. ${ }^{[6]}$ Recently, numerous studies have also demonstrated the multi-functionality of CNT yarns as fiber-shaped electrical sensors, actuators, supercapacitors, and energy harvesters (Figure 1). CNTs are carbon-based materials with a cylindrical nanostructure that can be envisaged as a layer of graphene rolled into a hollow cylinder. ${ }^{[7]}$ This structure is known as a single-walled nanotube, (SWNT). In fact, CNTs often exist as multiple concentric cylinders that are called multiwalled nanotubes (MWNTs). The latter are fabricated or "grown" by heating a precursor gas above $700^{\circ} \mathrm{C}$ so that vertically aligned and densely packed "forest" of MWNTs form on the solid substrate, such as mesoporous silica. ${ }^{[8]}$ In 2004, Zhang et al. established a major breakthrough by drawing sheets of MWNT taken from a nanotube forests and twisting these sheets to form yarn (Figure 2a). The CNT yarns exhibit excellent mechanical strength and have an electrical conductivity $~ 300$ S/cm at room temperature. ${ }^{[9]}$ Further refinements has improved the electrical conductivity of CNT yarn to $595 \mathrm{~S} / \mathrm{cm}$ at room temperature. ${ }^{[10]}$

Numerous studies have attempted to further improve the electrical conductivity of CNT yarns. For instance, the electrical conductivity can be regulated by inducing the oxidation on the surface of CNT yarn. In fact, oxidizing the CNT yarn in air or by the treatment of $\mathrm{HNO}_{3}$ enhanced its conductivity, whereas the conductivity was diminished by annealing CNT fibers

in $\mathrm{Ar}+6 \% \mathrm{H}_{2}$ at $800{ }^{\circ} \mathrm{C} .{ }^{[10]}$ Another approach has been to combine metal nanoparticles into 


\section{WILEY-VCH}

the CNT yarn. Au nanoparticle-coated CNT yarns improve the electrical conductivity, but these yarns also show a unique conductivity-temperature relationship unlike bare CNT yarn, resulting in semi-conductive behavior at temperature above $280 \mathrm{~K}$, whereas conductive behavior changed to metallic at temperature higher than $250 \mathrm{~K} .^{[10]}$

Other attempts showed that $\mathrm{Cu}$ - or Au- composite $\mathrm{CNT}$ yarns prepared by electrodeposition exhibit higher metal-like electrical conductivities $\left(2 \sim 3 \times 10^{5} \mathrm{~S} / \mathrm{cm}\right)$ compared to the bare CNT yarn $\left(5 \times 10^{2} \mathrm{~S} / \mathrm{cm}\right)$. However, their tensile strengths were reduced by around $30-50 \%{ }^{[11]}$ It has been further demonstrated that silver particle-coated CNT yarns improve the electrical conductivity $\left(4.28 \times 10^{4} \mathrm{~S} / \mathrm{cm}\right)$ without sacrificing its mechanical strength $^{[12]}$ and post-treatment of $\mathrm{CNT}$ yarn by coupling with $\mathrm{FeCl}_{3}$ enhances both the electrical conductivity $\left(3.5 \times 10^{3} \mathrm{~S} / \mathrm{cm}\right)$ and mechanical strength $(420 \mathrm{MPa}) \cdot{ }^{[13]}$ On the contrary, the encapsulation of CNT yarn by facile heat treatment has considerably improved both mechanical strength and thermal conductivity, but has also increased the electrical resistance. ${ }^{[14]}$

As an alternative approach, some research groups have tried to integrate various polymers with CNT yarns to improve electrical conductivity. It was shown that polyvinyl alcohol-inserted CNT yarn considerably enhances the electrical conductivity $\left(9.2 \times 10^{2} \mathrm{~S} / \mathrm{cm}\right)$, which is comparable to $\mathrm{HNO}_{3}$-induced oxidized and Au nanoparticle-coated CNT yarns. ${ }^{[15]} \mathrm{It}$ was also found that a conducting polymer, polypyrrole-contained CNT yarn shows a slightly higher electrical conductivity (around $2.60 \times 10^{2} \mathrm{~S} / \mathrm{cm}$ ) than pure CNT yarn $\left(2.15 \times 10^{2} \mathrm{~S} / \mathrm{cm}\right.$ ) in addition to improved mechanical strength. ${ }^{[16]}$ In contrast, a polyaniline SWCNT composite fiber made by electrospinning failed to greatly improve the electrical conductivity despite of good compatibility between two components. ${ }^{[17]}$ Interestingly, in situ observation by transmission electron microscopy during voltage application has found rotation and breakage of CNT yarn, suggesting that the damage can cause circuit failure due to the resistive heating 


\section{WILEY-VCH}

occurring during the current flow. ${ }^{[18]}$ Therefore, it seems that mechanical strength should also be considered when improving electrical conductivity.

CNT yarns of small diameter have exhibited good flexibility, while maintaining good strength and conductivity (Figure 2b). ${ }^{[19]}$ Silk-sheathed CNT yarns have been fabricated by electrospinning silk fibroin solution on rotating CNT yarns. ${ }^{[20]}$ Consequently, it has found that silk-sheathed CNT yarn exhibit a high mechanical strength, flexibility, durability, and electrical conductivity $\left(3.1 \times 10^{4} \mathrm{~S} / \mathrm{m}\right)$ suitable for textile electronics. ${ }^{[20]}$ However, CNT yarns are still limited in terms of stretchability. To overcome this limitation, remarkable improvements in the stretchability of CNT yarns has been achieved through structural deformations by introducing coiled structures by twisting the yarns under tension (Figure 2c-e). The coiled CNT yarns show spring-like extensibility while maintaining high electrical conductivity.

\section{CNT yarn as an electronic sensor}

There has been a growing demand for the development of smart monitoring systems to collect, quantify and qualify information associated with the human body. ${ }^{[21]}$ The monitoring systems are equipped with smart electronic sensors that are either worn externally or implanted into the body. ${ }^{[22]}$ Flexible fiber or yarn-based sensors are highly attractive because of their small diameters, high surface-to-volume ratios, light-weight, and suitability for integration into textiles. ${ }^{[23]}$ Since CNT yarns combine good mechanical and conductive properties, a variety of studies on CNT fiber or yarn as an electronic sensor have been actively investigated. Currently, most CNT fiber-composed strain, thermal, and potentiometric sensors are based on monitoring electrical resistance or capacitive changes of the CNT fiber in response to strain deformation, thermal change, or changes in chemical environment. In addition, some biochemical sensors operate by detecting current flow at a CNT electrode that is derived from oxidized molecules by low voltage input. 


\section{WILEY-VCH}

\subsection{Electronic strain sensor}

Electronic strain sensor can generate electrical signals, responding to strain deformation produced in response to an external force. Flexible and miniaturized strain sensors are expected to facilitate new approaches for smart wearable applications, such as the monitoring and detection for human motion, health, and medical treatment. ${ }^{[24]}$ Generally, the integration of conductive materials and flexible substrates is a common method to fabricate flexible strain sensors. The challenges for using CNT fibers or yarns as strain sensors has been to determine strain sensitive properties that can be monitored in situ and to enable high stretchability.

To verify intrinsic sensitivity of CNT yarn to strain, first studies investigated the variation in electrical resistance to tensile strain under variable temperature ranges. ${ }^{[25]}$ It was revealed that the resistance of CNT yarn changes in a linear manner when the strain increased from 0.5 to $1 \%$, and maintains a stable resistance at temperatures ranging from 77 to $373 \mathrm{~K} .{ }^{[25]}$ Another study has attempted to make entangled CNT yarn to utilize as an elastic strain sensor over large strain (up to 500\%). ${ }^{[26]}$ The entangled structure was fabricated by over-twisting to generate a snarled and knotted structures that reversibly and linearly changed in electrical resistance when untangled by pulling the yarn ends (tensile strain) (Figure 3a). ${ }^{[26]}$ These entangled CNT yarns have also generated the tensile actuation, simultaneously rotating a heavy object at high speed when released. ${ }^{[26]}$ It seems that the entangled CNT yarns could be used in some smart electronics which need physical movement (actuation) while sensing the strain deformation.

Applying elastic polymers to CNT yarns has also been used to increase the strain deformation. It has found that CNT-coated PU/cotton yarn exhibits a linear increase in electrical resistance to $300 \%$ strain, and could be reversible stretched and released about 


\section{WILEY-VCH}

300,000 times under $40 \%$ strain without damage (Figure $3 b$ ). ${ }^{[27]}$ In fact, the CNT coated PU/cotton strain sensor could effectively detect human motions such as finger movement, elbow motions, walking, and eye winking. ${ }^{[27]}$ Interestingly, Sim and colleagues have established a self-powered strain sensor based on PU/triboelectric materials/CNT composites (Figure 3c). ${ }^{[28]}$ Their device detected the voltage difference between a PU-wrapped positive triboelectric electrode and a CNT-coated negative triboelectric electrode when stretched and released. The voltage responses of the CNT composite strain sensor were detected as the applied strain increased from $10 \%$ to $50 \%$, and the sensor could be cycled around 10,000 times to $50 \%$ strain. ${ }^{[28]}$ Indeed, the self-powered strain sensor woven into the wristband of a glove effectively monitored the changes in the $\mathrm{x}-, \mathrm{y}$ - and diagonal directions. ${ }^{[28]}$ The piezoresistive concept has also been applied to develop strain gauges to detect piezoresistive changes in the arrangement of the CNT yarns by mechanical loading (Figure 3d). ${ }^{[29]}$ The piezoresistive CNT sensor has shown to reversibly detect the strain deformation. ${ }^{[29]}$ An interesting related device uses an array of several CNT yarns on grooved silicon to form a thermoacoustic chip (Figure 3e). ${ }^{[30]}$ The suspending CNT yarn arrays across the grooves linearly respond to sound pressure at $10,20,40 \mathrm{kHz}$, depending on the depth of the grooves. The acoustic sensor could be used as a microphone and was successfully integrated to earphones as an application in consumer electronics. ${ }^{[30]}$

\subsection{Electronic thermal and potentiometric sensors}

Temperature sensors can be classified into several types based on the material and their operation and include thermistors (temperature sensitive resistors) and thermocouples (voltage generating metal junctions) and silicon-based sensors. ${ }^{[2 b, 21 c, 31]}$ Many types of temperature sensors are used to detect the temperature by sensing changes in electrical resistance of the thermosensitive materials such as ceramics, polymers, and metals. ${ }^{[2 b, 21 c, 31]}$ 


\section{WILEY-VCH}

Importantly, new types of temperature sensor have attempted to monitor the body temperature in the real-time through the wearable sensor. ${ }^{[2 \mathrm{~b}, 21 \mathrm{c}, 31]}$

A limited number of studies have investigated CNT yarns as fiber-based temperature sensors that could be miniaturized and were sufficiently flexible for textile application. In 2008, one initial study has characterized the bridge current that is voltage drop across an external resistor, due to changing the temperature. The study revealed that the currents of a 4-ply CNT yarn were increased over a temperature range from -50 to $+50{ }^{\circ} \mathrm{C}$, suggesting the application as a temperature sensor. Further investigation has improved the temperature sensing capability, increasing the resistance change ratio of CNT by combining epoxy resin polymers. ${ }^{[32]}$ Other polymers have also been used. Sibinski and colleagues have produced flexible CNT based temperature sensor ${ }^{[33]}$ based on polyvinylidene fluoride (PVDF) monofilament due to its good flexibility and sufficient thermal resistance. The CNT-coated PVDF fibers exhibited a high positive temperature coefficient, indicating the resistance increased as temperature was raised. ${ }^{[33]}$

The potentionmetric sensor is a kind of chemical sensor that can determine the analytical concentration of target components. ${ }^{[34]}$ Currently, potentiometric sensors have received great attention due to their potential in a wide area, including environmental monitoring and medical diagnostics. ${ }^{[35]}$ For wearable applications it is preferable to integrate the potentionmetric sensors into textiles, so researchers have been developing fiber-based sensors including those based on CNTs.

In the potentionmetric sensor, output signals are generally characterized by the electrical potential difference of an electrode when compared to a reference electrode. As a potentionmetric sensor, it was first found that the construction of CNT-cotton yarn with a polymeric membrane could determine the concentration of $\mathrm{pH}, \mathrm{K}^{+}$, and $\mathrm{NH}_{4}{ }^{+}$as a fiber-shaped potentiometric sensor. ${ }^{[36]}$ A noticeable feature of CNT-cotton yarn was the stability of the 


\section{WILEY-VCH}

signals for the target ions, which can be attributed to the high capacitance of the CNTs. ${ }^{[36]}$ Also, CNT-cotton sensor covered a broad range of $\mathrm{pH}$ between 3 to 11, and limits of detection for other ions was $10 \mu \mathrm{M}$ for $\mathrm{K}^{+}$and $1 \mu \mathrm{M}$ for $\mathrm{NH}_{4}{ }^{+}$that are similar to values found for solid-state ion-selective electrodes. ${ }^{[36]}$

In summary, the current status has demonstrated the potential of CNT based sensors for temperature and chemical species. It is likely that more research will have to be continued to apply these materials in smart electronics such as portable and wearable devices.

\subsection{Electronic sensors for neurotransmitter monoamines}

Specialized chemical sensors are needed for detecting bioactive molecules. In central nervous system, for example, monoamines are a bioactive neurotransmitter consisting of one amino group attached to an aromatic ring by a two-carbon chain, which includes dopamine, serotonin, and epinephrine. ${ }^{[37]}$ Because the levels of monoamines are known to regulate the mood such as anxiety, and happiness, it is considered important to measure the monoamine levels in the blood. ${ }^{[38]}$

In biochemical analysis, fast-scan cyclic voltammetry (FSCV) is an electrochemical technique that can measure monoamine levels such as dopamine, serotonin, and norepinephrine. ${ }^{[39]}$ Since the monoamines can be oxidized by low voltage, the currents are

generated by electron transfer owing to the oxidation process by cyclic voltammetry. ${ }^{[39]}$ Carbon-based fibers are often used for microelectrodes because the carbon fiber electrodes are stable and inert at the voltages that is sufficient to oxidize the amine. ${ }^{[40]}$

Among carbon fibers, CNT yarns are attractive as a microelectrode substrate, and several studies have shown selectivity and sensitivity for monoamines. In 2013, Schmidt and colleagues have shown that CNT yarn electrodes could discriminate rapid fluctuations of dopamine and serotonin in acute brain slices. ${ }^{[41]}$ They have also demonstrated that the CNT 


\section{WILEY-VCH}

yarn microelectrode has a higher selectivity, sensitivity, and spatial resolution, as well as faster discrimination when compared to the conventional carbon fiber electrodes. ${ }^{[41]}$ In further detail analysis for high-speed measurement, it was revealed that CNT yarn microelectrodes could detect the changes in dopamine at the 2-ms time scale with FSCV, which indicates a 2-fold increase in speed than conventional carbon fiber electrodes. ${ }^{[42]}$ Also, CNT yarn electrode is suitable for detecting low concentration of dopamine to be $8 \mathrm{nM}$, and could measure the currents for dopamine oxidation to $25 \mu \mathrm{M} \cdot{ }^{[42]}$ Interestingly, laser-treated CNT yarn microelectrodes have further improved the temporal detection and sensitivity for in vivo dopamine due to the larger surface and higher oxygen content on the surface of the CNT yarn. ${ }^{[43]}$ Yang et al. have attempted to improve the sensitivity to dopamine, providing negatively charged surface by the treatment of chlorosulfonic acid in CNT yarn electrodes. ${ }^{[44]}$ Noticeably, the oxygen plasma etching and antistatic gun treatment of CNT yarn electrodes lead to 12-fold increase in the currents for dopamine. ${ }^{[45]}$ Further investigation has shown that the oxygen plasma etching augments surface oxygen content without the change of surface roughness, while the antistatic gun treatment facilitates surface roughness without the change in surface oxygen content. ${ }^{[45]}$ Other attempts to improve electrode surface characteristics have coated Nafion polymer after fabrication of CNT yarn onto a micro Pt-wire. This electrode has been shown to increase the selectivity and sensitivity to dopamine as a probe sensor. ${ }^{[46]}$ In summary, several studies have supported that CNT yarn electrodes are beneficial for high selective and sensitive detection for monoamines with fast-speed measurement.

\section{Electrochemical actuation of CNT yarn}

Advanced actuators that can operate without the need for manual interaction are gradually finding their way into smart electronic systems. ${ }^{[4]}$ Recent development in smart materials is encouraging miniaturized and multifunctional soft actuators that can be electrically 


\section{WILEY-VCH}

controlled. ${ }^{[48]}$ For the last several years, CNT yarns have been investigated as an artificial muscle, whose actuation may mimic physiological muscle. ${ }^{[49]}$ Those accumulated studies have demonstrated torsional and tensile actuation of CNT yarns by electrical, chemical, thermal or photonic power. ${ }^{[50]}$ The current review focuses specifically on electrically controlled CNT yarns.

As an artificial muscle, Foroughi and colleagues have first showed a reversible torsional actuation of CNT yarn in electrochemical system. ${ }^{[51]}$ The torsional actuation was $15,000^{\circ}$ rotations and 590 revolutions per minute, with the actuation mechanism due to the volume increase of CNT yarn by electrochemical double-layer charge injection. ${ }^{[51]}$ Further investigations have tried to utilize solid gel electrolyte-coated CNT yarn instead of liquid electrolyte, and consequently they showed all-solid-state torsional and tensile actuation of CNT yarn with no need for liquid solution. ${ }^{[52]}$ Such gel electrolyte based CNT yarn actuators are more suited to application in wearable devices and textile electronics.

Coiling of twisted fibers and yarns enables the torsional actuation in the twisted fiber to be translated to a tensile actuation along the length of the coil. Twisted and coiled CNT yarns have been electrochemically stimulated to generate tensile contractions. In one study, the CNT biscrolled with reduced graphene oxide have provided relatively large tensile actuation $(8.1 \%)$ in aqueous inorganic electrolytes, ${ }^{[53]}$ which has improved over 6-fold when compared to the previous results in an inorganic electrolyte $(1.3 \%) .{ }^{[54]}$ Most recently, Kim and colleagues have dramatically improved electrochemical-induced tensile stroke (15.1\%) and power capacity $(3.78 \mathrm{~kJ} / \mathrm{kg})$ of CNT yarn in an inorganic electrolyte by coiling plies of twist-released CNT yarns (Figure 4). ${ }^{[55]}$

Torsional and tensile actuation can also be stimulated by non-electrochemical means. Fast torsional actuation of CNT yarns by electrothermal stimulation has been achieved by infiltrating paraffin wax guest into yarns. ${ }^{[56]}$ Such paraffin waxes have the large volume 


\section{WILEY-VCH}

changes with thermal expansion. ${ }^{[56]}$ Thus, the applied electrical power lead to a high torsional and tensile actuation of paraffin-filled CNT yarn. ${ }^{[56]}$ These systems are electrolyte-free and fully dry. Interestingly, less dense coiled paraffin-infiltrated yarn has exhibited a different actuation mechanism where the melted paraffin waxes move out from yarns. ${ }^{[61]}{ }^{[57]}$ Some other guest polymers have been also been investigated for electrothermal actuation of CNT yarns. Polystyrene (PS) and poly(styrene-b-isoprene-b-styrene) (SIS) are known to induce specific volume change by phase transitions with increasing temperature. ${ }^{[58]}$ Accordingly, PS or SISinfiltrated CNT yarns have shown a significant volume expansion by applied electrical power, and PS-infiltrated CNT yarns $(29.7 \% \mathrm{~mm})$ have demonstrated much larger torsional behaviors than those of the SIS-infiltrated CNT yarn $\left(14.4^{\circ} / \mathrm{mm}\right) .{ }^{[59]}$ Recently, Song et al. have provided a large tensile stroke $(\sim 13.8 \%)$ within 5 sec by combining thermoplastic PU resin with CNT yarns. The electrothermal volume expansion of thermoplastic PU guest induced the contraction in length and untwisting of the CNT yarn, which returned to its original length when cooled. ${ }^{[60]}$

\section{Energy storage of CNT yarn}

Wearable electronic device enables a platform for monitoring various surrounding and physiological signals on the body. ${ }^{[61]}$ Consequently, wearable electronics require flexible energy storage system to maintain reliable performance and safety. ${ }^{[62]}$ Compared with batteries, supercapacitors can deal with unstable electrical input and output because electrical energy is regulated by charge, discharge, and recharge. ${ }^{[63]}$ Therefore, flexible supercapacitors are indispensable for wearable electronics.

The reliable transmission and distribution of electric charge in smart systems are required for efficient operation. Energy harvesting from variable sources and energy storage for later use is desirable and important in self-powered applications of sensing and actuating system. ${ }^{[64]}$ For example, the integration of supercapacitors with energy harvesters has been 


\section{WILEY-VCH}

developed as a self-charging power system to with high efficiency. ${ }^{[65]}$ Multifunctional materials capable of harvesting and/or with the capacity to store electrical energy are ideal for smart electronic systems. Numerous studies have demonstrated the remarkable capacitive performance of CNT yarns that make these materials stand out candidates in the field of supercapacitors. Some investigations have also investigated the functions of CNT yarns as an energy harvester. Those findings present a blueprint for enabling smart energy storage systems that are integrating both energy harvesting and storage through supercapacitance.

\subsection{Supercapacitor}

Capacitors are passive electronic components that stores electrical energy and consists of one or more pairs of conductors separated by an insulator. Devices that enable higher values of the energy density are called supercapacitors. ${ }^{[66]}$ With a raised demand for portable and nontoxic energy, electrochemical supercapacitors have been receiving great attention due to their higher power density and longer cyclic lifetime when compared with Li batteries. ${ }^{[67]}$ Unlike common capacitors, electrochemical supercapacitors depend on electrostatic and/or electrochemical principles for charge storage. Pseudo-capacitors store electrical energy by electron charge transfer between an electrode and electrolyte through fast surface redox reactions. ${ }^{[67]}$ The transition metal oxides, such as $\mathrm{MnO}_{2}$ and $\mathrm{NiO}_{2}$, are commonly used for redox reactions in pseudo-capacitors. Electrical double-layer capacitors (EDLCs) store electrical energy by ion adsorption at the interface between the porous surface of electrode and electrolytes and without any redox reaction. ${ }^{[67]}$ For EDLC electrodes, carbon-based materials have been frequently used due to their high porosity to facilitate increased ion adsorption. ${ }^{[68]}$ Comparing pseudo-capacitors and EDLCs, the former exhibit a higher energy density and shorter cyclic lifetimes, whereas EDLCs have somewhat limited energy density and longer 


\section{WILEY-VCH}

cyclic lifetimes. Hence, functional materials with pseudo-capaciticity have attempted to integrate to EDLCs to improve the performance of the supercapacitor.

\subsubsection{Flexible CNT yarn supercapacitor}

The introduction of pseudocapacitance materials such as conducting polymers has known to improve the energy density of supercapacitors. Many researchers have tried to incorporate various conducting polymers or metals to improve energy density of CNTs. Initial studies used a conducting polymer, poly(3,4-ethylenedioxythiophene) (PEDOT) coated on CNT sheet, which has provided micro-supercapacitor yarns with $30 \mu \mathrm{m}$ diameter by twisting. ${ }^{69]}$ As a supercapacitor, the two-ply PEDOT composite CNT yarns have exhibited volumetric energy density $\left(70 \mathrm{mWh} / \mathrm{cm}^{3}\right)$ and power density $\left(7910 \mathrm{~W} / \mathrm{cm}^{3}\right)$ with high mechanical strength and flexibility. ${ }^{[69]}$ Wang and colleagues have infiltrated polyaniline nanowire into CNT yarns. ${ }^{[63]}$ The symmetric polyaniline composite CNT yarn supercapacitor has exhibited an areal capacitance of $38 \mathrm{mF} / \mathrm{cm}^{2}$, which has been able to co-woven with conventional cotton yarns, suggesting potential application to wearable electronics. ${ }^{[63]}$ Further investigation has improved the electrical capacitance, strength and efficiency of polyaniline composite CNT yarns by using oxidized CNT yarns through a gamma irradiation treatment that increased the concentration of carboxyl groups on $\mathrm{CNT} .{ }^{[70]}$

To increase intrinsic capacitance using pseudo-capacitive materials, the use of transition metal oxides such as $\mathrm{NiO}_{2}, \mathrm{Co}_{3} \mathrm{O}_{4}$, and $\mathrm{MnO}_{2}$ has been investigated. ${ }^{[71]}$ The application of $\mathrm{MnO}_{2}$ in supercapacitors is highly attractive because of the material's excellent

capacitance, low cost, and environmental compatibilities. ${ }^{[72]}$ However, $\mathrm{MnO}_{2}$ still has a disadvantage in poor electrical conductivity and brittleness in the application of supercapacitors. One solution is to deposit $\mathrm{MnO}_{2}$ onto carbon-based materials with high electrical conductivity 


\section{WILEY-VCH}

to compensate the weakness of $\mathrm{MnO}_{2}$, and consequently it has been promoting the energy density of supercapacitors. ${ }^{[73]}$

Considering fiber or threadlike supercapacitors for wearable electronics, $\mathrm{MnO}_{2}$ composite CNT yarns have been developed as a supercapacitor electrode. In 2014, Choi et al. have provided a flexible supercapacitor with $\mathrm{MnO}_{2}$ particles deposited on the surface of a twisted CNT yarn with high internal porosity. ${ }^{[74]}$ The deposited $\mathrm{MnO}_{2}$ is trapped in the pores of aligned CNT bundles, and provides an enlarged electrolytic surface area of $\mathrm{MnO}_{2} \cdot{ }^{[81]} \mathrm{As} \mathrm{a}$ result, the symmetric $\mathrm{MnO}_{2}$-deposited CNT yarns showed a high capacitance of $25.4 \mathrm{~F} / \mathrm{cm}^{3}$ and a power density of $127 \mathrm{~mW} / \mathrm{cm}^{3}$ in $\mathrm{KOH}$ gel electrolyte. ${ }^{[74]}$ Further investigation demonstrated that the charge storage capability could be improved by alternating deposition of $\mathrm{MnO}_{2}$ and $\mathrm{Ag}$ layers. ${ }^{[75]}$ The conductive Ag particles reduce the solid-state charge diffusion length in the $\mathrm{MnO}_{2}$, and also collect the generated electrons during the charge and discharge processes. ${ }^{[75]}$ Another research group has also developed an asymmetric two-ply CNT yarn supercapacitor. ${ }^{[76]}$ It was composed of as-spun CNT yarn as negative electrode and $\mathrm{MnO}_{2}$ composite CNT yarn as positive electrode. ${ }^{[76]}$ Consequently, the asymmetric two-ply CNT yarn supercapacitor achieved a power density of $19250 \mathrm{~W} / \mathrm{kg}$ in $\mathrm{H}_{3} \mathrm{PO}_{4}$ gel electrolye. ${ }^{[76]}$ Symmetric $\mathrm{Co}_{3} \mathrm{O}_{4}$ deposited CNT yarn supercapacitors have a areal capacitance of $52.6 \mathrm{mF} / \mathrm{cm}^{2}$ and energy density of $1.10 \mu \mathrm{Wh} / \mathrm{cm}^{3}$ in $\mathrm{H}_{2} \mathrm{SO}_{4}$ gel electrolyte. ${ }^{[77]}$ These studies have shown that the psuedocapacitive material needs to be incorporated as fine powder and thin films because thick layers can hamper areal and volumetric capacitances due to intrinsic low electrical conductivity. ${ }^{[78]}$

The transition metal MXene has created interests a conducting guest material to be used in various areas of supercapacitor. ${ }^{[79]}$ The MXenes are a new conducting material with high electrical conductivity $(\sim 9880 \mathrm{~S} / \mathrm{cm})$ and hydrophobic surface, which is composed of carbides, nitrides, or carbonitrides. ${ }^{[80]}$ As a capacitor, it has been revealed that the 


\section{WILEY-VCH}

intercalation of cations into layered surface of hydrophilic $\mathrm{Ti}_{3} \mathrm{C}_{2}$ Mxene provides excellent volumetric capacitance of $\sim 1500 \mathrm{~F} / \mathrm{cm}^{3} \cdot{ }^{[81]}$ However, MXene-based fiber or yarn showed a limited performance in energy capacitance due to relatively low MXene loading and reduced electrical conductivity that was introduced during the fabricating process from MXene nanosheet. ${ }^{[82]}$ For this reason, Wang and colleagues have attempted to develop Mxenebiscrolled CNT yarn with up to 98 wt\% loading. ${ }^{[83]}$ The symmetric (Mxene/CNT and Mxene/CNT) electrode configuration has resulted in an areal capacitance of $3188 \mathrm{mF} / \mathrm{cm}^{2}$ and volumetric capacitance of $1083 \mathrm{~F} / \mathrm{cm}^{3}$ in $\mathrm{H}_{2} \mathrm{SO}_{4}$ electrolyte. ${ }^{[83]}$ Moreover, the asymmetric configuration $\left(\mathrm{RuO}_{2} / \mathrm{CNT}\right.$ and Mxene/CNT) has showed a maximum energy density (61.6 $\mathrm{mWh} / \mathrm{cm}^{3}$ ) and power density $\left(5428 \mathrm{~mW} / \mathrm{cm}^{3}\right)$, which has powered small electronic devices such as a distal watch and timer. ${ }^{[83]}$

A current metal collector has been applied into the linear supercapacitor to efficiently transport produced charges along the length of the supercapacitor. In the two-ply yarn supercapacitors, the metal filament core operatings as a current collector have been known to assist the movement of electrical charges, resulting to improve storage capacities in the energy and power. For instance, when single hybrid yarn was further plied with a metal wire, it had considerably improved the volumetric capacitance up to $179 \mathrm{~F} / \mathrm{cm}^{3}$ with ultrafast charge and discharge. ${ }^{[84]}$ CNT yarns have been twisted on a conductive Pt metal filament core for long, strong, and flexible threadlike supercapacitor. ${ }^{[85]}$ Consequently, the symmetric CNT-twisted Pt supercapacitor has provided a gravimetric capacitance of $86.2 \mathrm{~F} / \mathrm{g}$, an areal capacitance of 52.5 $\mathrm{mF} / \mathrm{cm}^{2}$, an equivalent capacitance of $241.3 \mu \mathrm{F} / \mathrm{cm}$ in phosphoric acid gel electrolyte. ${ }^{[85]}$ To find out the most effective current collector for CNT yarn, Zhang et al. have attempted to compare several candidate metal filaments, including $\mathrm{Pt}, \mathrm{Au}, \mathrm{Ag}, \mathrm{AuAg}, \mathrm{PtCu}$, and $\mathrm{Cu}^{[86]} \mathrm{As}$ a result, $\mathrm{Cu}$ and $\mathrm{PtCu}$ metal filaments have exhibited better capacitance than the other four metal filaments. ${ }^{[86]}$ They have further observed that $\mathrm{Cu}$-contained filament is only oxidized 


\section{WILEY-VCH}

on its surface. ${ }^{[86]}$ Thus, they have suggested that unoxidized core portion of $\mathrm{Cu}$ can play the role as current collector due to high conductivity, and oxidized $\mathrm{Cu}$ on the surface also can function as active guest material. ${ }^{[86]}$

CNT can also be fabricated into diverse fiber shapes and include other active materials. For example, chitosan composite CNT fibers have been obtained through wet-spinning technique and investigated as a micro-supercapacitor. The wet-spinning has enabled various hollow, twisted, and ribbon shaped fibers composed of graphene and SWNT composite. ${ }^{[87]}$ Among them, ribbon fiber showed higher electrical conductivity $(524 \mathrm{~S} / \mathrm{cm})$ and areal capacitance $\left(2.38 \mathrm{mF} / \mathrm{cm}^{2}\right)$ than those of other shapes in $\mathrm{LiCl}$ gel electrolyte. ${ }^{[87]}$ Furthermore, Kou et al. have developed core-sheath fiber composed of graphene/CNT core and electrolyte sheath by coaxial wet-spinning method. ${ }^{[88]}$ The core-sheath supercapacitor has exerted the areal capacitance of $177 \mathrm{mF} / \mathrm{cm}^{2}$ and energy density of $3.84 \mu \mathrm{F} / \mathrm{cm}$ in $\mathrm{H}_{3} \mathrm{PO}_{4}-\mathrm{PVA}$ gel electrolyte. ${ }^{[88]}$

\subsubsection{Stretchable CNT yarn supercapacitor}

Early investigations have attempted to increase the stretchability of CNT film or forest by the combination with rubber-like polymers. A rubber-like stretchable hybrid CNT film has been provided by coating uniformly dispersed SWNTs with PDMS-based rubber, resulting in high conductivity $(57 \mathrm{~S} / \mathrm{cm})$ and stretchability (134\% elastic deformation). ${ }^{[89]}$ In a slightly easier way, direct infiltration of the aligned arrays of CNTs (CNT forests) with a PU solution has produced a high elastomeric conductive CNT composite. ${ }^{[90]}$

As an alternative approach flexible fiber-shaped CNT supercapacitors have been achieved by coiling the elastic polymer composite CNT fiber or yarns. This coiling in fibers or yarns can provide structural stretchability. ${ }^{[56,91]}$ In one instance, the electrode of a highly stretchable supercapacitor has been developed by a coiled $\mathrm{MnO}_{2}$-deposited nylon/CNT fiber (Figure 5a). ${ }^{[92]}$ The parallel symmetrical a coiled $\mathrm{MnO}_{2}$-deposited nylon/CNT fiber in solid- 


\section{WILEY-VCH}

state reversibly stretched by $150 \%$ strain with only a small reduction in capacitance (15\%), and retained the capacitance during a stretch-release cycle. ${ }^{[92]}$ The areal capacitance of this system was $40.9 \mathrm{mF} / \mathrm{cm}^{2}$ and power density of $66.9 \mathrm{~mW} / \mathrm{cm}^{2} \cdot{ }^{[92]}$ Further investigation has improved the performance of elastomeric supercapacitor by a coiled $\mathrm{MnO}_{2}$-biscrolled CNT yarn (Figure 5b). ${ }^{[93]}$ The biscrolling process has lead to a higher loading of $\mathrm{MnO}_{2}$ nanoparticles (above 90 $\mathrm{wt} \%$ ), and a coiling process has again produced a flexible and stretchable fiber despite the high loading of brittle metal oxide. ${ }^{[93]}$ In functional performance, the maximum areal capacitance of a coiled $\mathrm{MnO}_{2}$-biscrolled CNT yarn was 100 -fold higher than the previously developed coiled $\mathrm{MnO}_{2}$-deposited nylon/CNT fiber. ${ }^{[93]}$ Interestingly, Choi and colleagues have developed the structure of twistable and stretchable sandwich fiber for sensor and supercapacitors (Figure 5c). ${ }^{[94]}$ To fabricate the structure, fiber-shaped rectangular rubber has been first stretched in the axial direction by $300 \%$ strain, and then CNT sheets have been covered on opposite sides of the stretched rectangular core rubber. ${ }^{[94]}$ When the pre- stretched fiber was relaxed from the strain, the CNT sheets on the rubber surface forms microscopically bulked configuration where the CNT coating becomes folded. This structure could contribute a large electrochemical surface area. ${ }^{[94]}$ Consequently, two symmetrical buckled CNT fibers has provided a high areal capacitance of $11.88 \mathrm{mF} / \mathrm{cm}^{2}$ and retention of over $95 \%$ of initial energy storage capability on large strain deformations in $\mathrm{LiCl}$ gel electrolyte. ${ }^{[94]}$

\subsection{Energy harvesters}

It is often highlighted how useful it would be to convert low level 'waste' energies into electrical energy and such systems are called "energy harvesting technologies". Various energy harvesters have been developed and are based on the target energy source, such as thermal, solar, or mechanical energies. ${ }^{[95]}$ Energy harvesting from available energy sources could enable self-powered operation in micro-robots, soft robots, implantable devices, and 


\section{WILEY-VCH}

wearable devices. ${ }^{[64]}$ To date, there has been active investigations into the electrical energy harvesting by CNT-based fibers or yarns from thermal, photo, and mechanical energies.

\subsubsection{Thermoelectric conversion using CNT yarns}

Thermoelectric generators are designed to convert heat flow directly into electrical energy with recent research focusing on systems that can efficiently harvest electrical energy from small temperature fluctuations. Certain polymer composite CNT films has known to exhibit thermoelectric effects that are capable of the conversion between thermal and electrical energy. ${ }^{[96]}$ As a representative example of these composite CNT films, an alternative stack of conducting layer (PVDF composite CNT film) and insulation layer are known to produce thermoelectric voltages. ${ }^{[96 a]}$ However, those film-based thermoelectric generators are somewhat limited for wearable and portable electronics. For this reason, some researchers have developed fiber-shaped thermoelectric harvesters. Using electrospinning, the nanofibers have been coated with $n$ - and p-type semiconductor sheaths $\left(\mathrm{Bi}_{2} \mathrm{Te}_{3}\right.$ and $\mathrm{Sb}_{2} \mathrm{Te}_{3}$, respectively), and then twisted into flexible yarns. ${ }^{[97]}$ Finally, thermoelectric textile has been fabricated by alternatively incorporating $n$ - and p-type yarns into textile, providing electrical energy between segments of the n- and p-type yarns from thermopowers. ${ }^{[97]}$ Choi et al. have presented a flexible thermoelectric generator based on CNT yarns without metal electrodes. ${ }^{[98]}$ The CNT yarn was alternatively doped into n-type with polyethylenimine and p-type with $\mathrm{FeCl}_{3}$, and additional CNT yarns used as electrodes between n-type and p-type to minimize the circuit resistance (Figure 6a) ${ }^{[98]}$ The CNT-based thermoelectric generator (60 pairs of n- and p-doped CNT yarn) has exhibited the maximum power density of 10.85 and $697 \mu \mathrm{W} / \mathrm{g}$ at temperature difference of only 5 and $40 \mathrm{~K}$, respectively. ${ }^{[98]}$

\subsubsection{Photoelectric conversion of CNT yarns}




\section{WILEY-VCH}

Fiber-shaped solar cells are applicable for flexible electronics and designed for solar energy harvesting. For high power conversion efficiencies of CNT-based solar cells, Pt film or particles has been widely used as a composite material in electrodes for electrochemical energy conversion. ${ }^{[99]}$ However, the deposited Pt particles tend to aggregate on the fiber and often render the fiber brittle when over-loaded, which causes lower energy transition efficiency. ${ }^{[100]}$ To improve stability and flexibility, a fiber-shaped CNT-based solar cell has been produced by twisting Pt-adsorbed CNT yarns around $\mathrm{TiO}_{2}$-based counter electrode (Figure $6 \mathrm{~b}$ ). ${ }^{[101]}$ This Pt nanoparticle- loaded solar cell has exhibited a similar power conversion efficiency of $4.85 \%$ under standard illumination $\left(100 \mathrm{~mW} / \mathrm{cm}^{2}\right)$, compared with the Pt wire composite CNT solar cells $(4.23 \%) \cdot{ }^{[101]}$ In addition, the efficiency of CNT solar cell was stable during repeated bending to $180^{\circ}$ angle and straightening for 500 cycles. ${ }^{[101]}$ In similar work, CNT yarn has been twisted around a conducting polymer-coated counter electrode, providing power conversion efficiency in the range $1.4 \%$ to $2.3 \% .{ }^{[102]}$ Interestingly, Yu et al. have attempted a photoelectric conversion by photochemical actuation of CNT yarns. ${ }^{[103]}$ The photoelectric CNT device was composed of two functional electrodes; one electrode was prepared by CNT yarn coated with poly(tetrafluoroethylene) (PTFE) to store charges. The second electrode was made with the bilayer of CNT/paraffin wax/polyimide composite strip for photochemical bending. The photoelectric CNT device was reversibly actuated in response to periodical irradiation of visible light, generating output a peak voltage of around $150 \mathrm{mV} .^{[103]}$

\subsubsection{Mechanoelectric conversion of CNT yarns}

All moving parts carry mechanical energy, which highlights the potential of mechanoelectric conversion for powering electronics especially in robotics and wearable technologies. Currently, a variety of studies have presented energy harvesting based on piezoelectric and triboelectric technologies that generate voltages from mechanical 


\section{WILEY-VCH}

deformations. ${ }^{[104]}$ However, power generation from these systems is limited to high-frequency and low-strain deformations for efficient performance due to the lack of their elasticity. ${ }^{[104]}$ Moreover, piezoelectric and triboelectric harvesters cannot be easy deployed in aqueous environments. ${ }^{[104]}$ On the other hand, fiber-shaped EDLC harvesters are known to be sensitive to low frequencies and high strain deformations due to their high flexibility and stretchability. In addition, these systems operate electrochemically and are compatible with aqueous environments.

Recent breakthrough research has provided a new technology for converting mechanical energy to electrical energy by using stretch-induced capacitance changes of coiled CNT yarns. ${ }^{[105]}$ Without the need for an externally-applied bias voltage, a coiled CNT yarn and counter electrode electrochemically provides electrical energy when stretched (Figure 6c). ${ }^{[105]}$ Because electrochemical capacitance changes due to the tensile stroke or torsional rotation drive electrical energy, these harvesters have been dubbed a 'Twistron' harvester. As applications, Twistron harvester that is sewn into a shirt can be used for monitoring breathing, and also could harvest the energy of near-shore ocean waves in sea water. ${ }^{[105]}$

\section{Conclusion and Future Perspectives}

Well known for their high electrical conductivity and mechanical strength, CNTs also display useful electrochemical properties and, combined, these attributes are leading to new types of sensors, actuators and energy storage devices in integrated smart electronic systems (Figure 1). In addition, the recent development of CNT yarns and their conversion to highly stretchable materials by coiled is accelerating their functional applications in emerging flexible and wearable devices. Despite these rapid advances, there is still a need to further improve material properties and enhance device performance by fabricating prototypes with integrated smart materials. 


\section{WILEY-VCH}

CNT-fiber based sensors have provided functions as strain sensors, potentiometric sensors, and electrochemical biosensors. The miniaturized and robust (flexible / stretchable) CNT yarn sensors have great potential for application as wearable, portable, and implantable electronics. Importantly, the recent finding of a coiled CNT yarn harvester to convert mechanical energy into electrical energy suggests the possibility of self-powered sensors to monitor structural deformation, environmental conditions and human health. Combining an energy harvester with sensors opens the prospect of powering smart sensors through strain deformations underlying human motions, mechanical vibrations or fluid flow. These selfpowered sensors have a great advantage of being deployable for long periods in difficult places where battery exchange is difficult. Implantable electronic devices are a prime example.

As an electronic actuator, CNT yarns and/or CNT composite yarns are known to exhibit torsional and/or tensile actuation in response to diverse stimulus, including electrochemical, thermal, photonic and by absorption/desorption of small molecules. In particular, the coiling and twisting the CNT yarn fibers further provides increased strength and actuation comparable to those of physiological muscle. These fiber-based actuators are ideally suited to smart textiles application where wearer comfort can be dynamically tuned using mechanically actuating fibers. Fabrics that change their porosity to trap or release heat and / or humidity on demand is one application example. One area of further work for CNT actuators is the desire to improve their energy conversion efficiency. The best performance to date is the $5.4 \%$ conversion (a contractile energy efficiency) of input electrical energy to output mechanical energy in an electrochemically operated coiled CNT yarn. ${ }^{[52 b]}$

As an electronic energy storage, CNT-based fiber supercapacitors exhibit superior performance with the added advantages of flexibility and stretchability, which are important in wearable and portable electronics. As with self-powered sensors, the opportunity now exists to couple CNT energy harvesting with energy storage. As mentioned earlier, CNT yarns also 


\section{WILEY-VCH}

function as energy harvester through thermoelectric, photoelectric, and mechanoelectric conversions. In many applications, the energy drain will be intermittent (eg. occasional sensing, actuation or data transmission). Energy harvesting, however, can be continuous but requires efficient storage for later use. Further work is needed to develop such combined devices and to improve the energy harvesting efficiency from low energy sources, such as small temperature gradients, low frequency vibrations and low light conditions.

Overall, CNT yarns and CNT-based fibers have many advantages for application as sensors, actuators, and energy harvesters as mentioned above. However, CNT yarns also have some limitations in performance. The need for electrolyte-filled devices also requires additional packaging that can add weight and bulk to the device. Furthermore, the potential for adverse human health effects associated with CNTs is still debated and applications require rigorous testing both during use and during the fabrication of the CNT based devices. Finally, it is likely that mass production or scale-up is somewhat limited since the unit cost of production of CNT yarns is still high.

In summarizing, much recent works demonstrate that CNT yarns or its composites are valuable materials with great potential for smart devices. Their functions cover sensing, actuation, and energy storage/harvesting and can be formed into flexible, stretchable, portable, wearable, and/or implantable high quality devices. In addition, this smart system is also required to robotic system. Recently, there has been growing in the interest of soft and deformable structures in the robotic system. ${ }^{[48,106]}$ These robotic devices can safely interface with the human body, and adapt to unpredictable and inapplicable environments in which conventional rigid robots are not viable. ${ }^{[107]}$ However, it is not fully understood how to achieve sensing, actuation, and integration in soft robots. ${ }^{[108]}$ In light of this point, CNT yarns could be applicable in the overall field for sensing, actuating, and energy storage in soft robotic systems. 


\section{WILEY-VCH}

In particular, functional integration of energy harvester and supercapacitance of CNT yarns might enable soft robotic to monitor and move with no need for electric motor and battery.

\section{Acknowledgements}

The research was supported by the Creative Research Initiative Center for Selfpowered Actuation of the National Research Foundation of Korea (NRF) in Korea.

\section{Conflict of Interest}

The authors declare no conflict of interest. 


\section{References}

[1] B. W. An, J. H. Shin, S. Y. Kim, J. Kim, S. Ji, J. Park, Y. Lee, J. Jang, Y. G. Park, E. Cho, S. Jo, J. U. Park, Polymers (Basel) 2017, 9.

[2] a) X. Wang, Z. Liu, T. Zhang, Small 2017, 13; b) A. Servati, L. Zou, Z. J. Wang, F. Ko, P. Servati, Sensors (Basel) 2017, 17.

[3] a) Z. Hao, Y. Pan, W. Shao, Q. Lin, X. Zhao, Biosens. Bioelectron. 2019, 134, 16; b) M. Cai, S. Nie, Y. Du, C. Wang, J. Song, ACS Appl. Mater. Interfaces 2019, 11, 14340; c) S. Wang, T. Chinnasamy, M. A. Lifson, F. Inci, U. Demirci, Trends Biotechnol. 2016, 34, 909; d) X. Wang, L. Dong, H. Zhang, R. Yu, C. Pan, Z. L. Wang, Adv. Sci. (Weinh) 2015, 2, 1500169.

[4] a) H. C. Kim, S. Mun, H. U. Ko, L. Zhai, A. Kafy, J. Kim, Smart Mater. Struct. 2016, 25; b) Y. Yang, Y. Chen, Y. Li, M. Z. Q. Chen, Y. Wei, Soft Robot 2017, 4, 147; c) R. Verma, R. R. Adhikary, R. Banerjee, Lab Chip 2016, 16, 1978; d) C. Choi, Y. Lee, K. W. Cho, J. H. Koo, D. H. Kim, Acc. Chem. Res. 2019, 52, 73.

[5] a) Y. Chen, B. Xu, J. Gong, J. Wen, T. Hua, C. W. Kan, J. Deng, ACS Appl. Mater. Interfaces 2019, 11, 2120; b) J. S. Heo, J. Eom, Y. H. Kim, S. K. Park, Small 2018, 14.

[6] a) V. R. Raphey, T. K. Henna, K. P. Nivitha, P. Mufeedha, C. Sabu, K. Pramod, Mater. Sci. Eng. C Mater. Biol. Appl. 2019, 100, 616; b) R. H. Baughman, A. A. Zakhidov, W. A. de Heer, Science 2002, 297, 787.

[7] S. Iijima, Nature 1991, 354, 56.

[8] Z. F. Ren, Z. P. Huang, J. W. Xu, J. H. Wang, P. Bush, M. P. Siegal, P. N. Provencio, Science 1998, 282, 1105.

[9] M. Zhang, K. R. Atkinson, R. H. Baughman, Science 2004, 306, 1358.

[10] Q. W. Li, Y. Li, X. F. Zhang, S. B. Chikkannanavar, Y. H. Zhao, A. M. Dangelewicz, L. X. Zheng, S. K. Doorn, Q. X. Jia, D. E. Peterson, P. N. Arendt, Y. T. Zhu, Adv. Mater. 2007, 19, 3358.

[11] L. K. Randeniya, A. Bendavid, P. J. Martin, C. D. Tran, Small 2010, 6, 1806.

[12] D. Zhang, Y. Zhang, M. Miao, Nanotechnology 2014, 25, 275702.

[13] Y. M. Choi, H. Choo, H. Yeo, N. H. You, D. S. Lee, B. C. Ku, H. C. Kim, P. H. Bong, Y. Jeong, M. Goh, ACS Appl. Mater. Interfaces 2013, 5, 7726.

[14] M. G. Hahm, J. H. Lee, A. H. Hart, S. M. Song, J. Nam, H. Y. Jung, D. P. Hashim, B. Li, T. N. Narayanan, C. D. Park, Y. Zhao, R. Vajtai, Y. A. Kim, T. Hayashi, B. C. Ku, M. Endo, E. Barrera, Y. J. Jung, E. L. Thomas, P. M. Ajayan, ACS Nano 2013, 7, 10971.

[15] K. Liu, Y. Sun, X. Lin, R. Zhou, J. Wang, S. Fan, K. Jiang, ACS Nano 2010, 4, 5827.

[16] J. Foroughi, G. M. Spinks, S. R. Ghorbani, M. E. Kozlov, F. Safaei, G. Peleckis, G. G. Wallace, R. H. Baughman, Nanoscale 2012, 4, 940.

[17] M. S. Kang, M. K. Shin, Y. A. Ismail, S. R. Shin, S. I. Kim, H. Kim, H. Lee, S. J. Kim, Nanotechnology 2009, 20, 085701.

[18] T. Tokunaga, Y. Hayashi, T. Iijima, Y. Uesugi, M. Unten, K. Sasaki, T. Yamamoto, Micron 2015, 74, 30.

[19] a) X. B. Zhang, K. L. Jiang, C. Teng, P. Liu, L. Zhang, J. Kong, T. H. Zhang, Q. Q. Li, S. S. Fan, Adv. Mater. 2006, 18, 1505; b) K. Jiang, Q. Li, S. Fan, Nature 2002, 419, 801.

[20] Z. Yin, M. Jian, C. Wang, K. Xia, Z. Liu, Q. Wang, M. Zhang, H. Wang, X. Liang, X. Liang, Y. Long, X. Yu, Y. Zhang, Nano Lett. 2018, 18, 7085.

[21] a) D. Dias, J. Paulo Silva Cunha, Sensors (Basel) 2018, 18; b) D. Baumgartel, C. Mielke, R. Haux, Stud. Health. Technol. Inform. 2018, 247, 476; c) Q. Li, L. N. Zhang, X. M. Tao, X. Ding, Adv. Healthc. Mater. 2017, 6. 
[22] a) P. J. Rivero, J. Goicoechea, F. J. Arregui, Sensors (Basel) 2019, 19; b) M. Liu, X. $\mathrm{Pu}$, C. Jiang, T. Liu, X. Huang, L. Chen, C. Du, J. Sun, W. Hu, Z. L. Wang, Adv. Mater. 2017, 29.

[23] a) R. Tabassum, B. D. Gupta, Analyst 2015, 140, 1863; b) S. H. Kim, J. J. Lee, D. C. Seo, J. O. Lim, Int. J. Mod. Phys. B 2003, 17, 1368.

[24] a) L. Liu, T. J. Webster, Curr. Osteoporos. Rep. 2016, 14, 386; b) W. C. Chuang, H. T. Lin, W. L. Chen, Sensors (Basel) 2015, 15, 3975; c) F. Yin, H. Lu, H. Pan, H. Ji, S. Pei, H. Liu, J. Huang, J. Gu, M. Li, J. Wei, Sci. Rep. 2019, 9, 2403.

[25] H. Zhao, Y. Zhang, P. D. Bradford, Q. Zhou, Q. Jia, F. G. Yuan, Y. Zhu, Nanotechnology 2010, 21, 305502.

[26] Y. Li, Y. Shang, X. He, Q. Peng, S. Du, E. Shi, S. Wu, Z. Li, P. Li, A. Cao, ACS Nano 2013, 7, 8128.

[27] Z. Wang, Y. Huang, J. Sun, Y. Huang, H. Hu, R. Jiang, W. Gai, G. Li, C. Zhi, ACS Appl. Mater. Interfaces 2016, 8, 24837.

[28] H. J. Sim, C. Choi, S. H. Kim, K. M. Kim, C. J. Lee, Y. T. Kim, X. Lepro, R. H. Baughman, S. J. Kim, Sci. Rep. 2016, 6, 35153.

[29] J. L. Abot, M. R. Gongora-Rubio, J. C. Anike, C. Y. Kiyono, L. A. M. Mello, V. F. Cardoso, R. L. S. Rosa, D. A. Kuebler, G. E. Brodeur, A. H. Alotaibi, M. P. Coene, L. M. Coene, E. Jean, R. C. Santiago, F. H. A. Oliveira, R. Rangel, G. P. Thomas, K. Belay, L. W. da Silva, R. T. Moura, A. C. Seabra, E. C. N. Silva, Sensors (Basel) 2018, 18.

[30] Y. Wei, X. Lin, K. Jiang, P. Liu, Q. Li, S. Fan, Nano Lett. 2013, 13, 4795.

[31] a) S. Khan, S. Ali, A. Bermak, Sensors (Basel) 2019, 19; b) M. Ramakrishnan, G. Rajan, Y. Semenova, G. Farrell, Sensors (Basel) 2016, 16.

[32] S. Gong, Z. H. Zhu, Z. Li, Phys. Chem. Chem. Phys. 2017, 19, 5113.

[33] M. Sibinski, M. Jakubowska, M. Sloma, Sensors (Basel) 2010, 10, 7934.

[34] R. Rahimi, M. Ochoa, A. Tamayol, S. Khalili, A. Khademhosseini, B. Ziaie, ACS Appl. Mater. Interfaces 2017, 9, 9015.

[35] a) M. Cuartero, M. Parrilla, G. A. Crespo, Sensors (Basel) 2019, 19; b) P. Ciosek, W. Wroblewski, Sensors (Basel) 2011, 11, 4688.

[36] T. Guinovart, M. Parrilla, G. A. Crespo, F. X. Rius, F. J. Andrade, Analyst 2013, 138, 5208.

[37] M. A. Kurian, P. Gissen, M. Smith, S. Heales, Jr., P. T. Clayton, Lancet Neurol. 2011, $10,721$.

[38] J. Ng, A. Papandreou, S. J. Heales, M. A. Kurian, Nat. Rev. Neurol. 2015, 11, 567.

[39] C. E. John, S. R. Jones, in Electrochemical Methods for Neuroscience (Eds: A. C. Michael, L. M. Borland), Boca Raton (FL) 2007.

[40] R. M. Wightman, Science 2006, 311, 1570.

[41] A. C. Schmidt, X. Wang, Y. Zhu, L. A. Sombers, ACS Nano 2013, 7, 7864.

[42] C. B. Jacobs, I. N. Ivanov, M. D. Nguyen, A. G. Zestos, B. J. Venton, Anal. Chem. 2014, 86, 5721.

[43] C. Yang, E. Trikantzopoulos, M. D. Nguyen, C. B. Jacobs, Y. Wang, M. MahjouriSamani, I. N. Ivanov, B. J. Venton, ACS Sens. 2016, 1, 508.

[44] C. Yang, E. Trikantzopoulos, C. B. Jacobs, B. J. Venton, Anal. Chim. Acta. 2017, 965, 1.

[45] C. Yang, Y. Wang, C. B. Jacobs, I. N. Ivanov, B. J. Venton, Anal. Chem. 2017, 89, 5605.

[46] W. Al-Graiti, Z. Yue, J. Foroughi, X. F. Huang, G. Wallace, R. Baughman, J. Chen, Sensors (Basel) 2017, 17. 
[47] S. Poppinga, C. Zollfrank, O. Prucker, J. Ruhe, A. Menges, T. Cheng, T. Speck, Adv. Mater. 2018, 30, e1703653.

[48] L. Hines, K. Petersen, G. Z. Lum, M. Sitti, Adv. Mater. 2017, 29.

[49] S. M. Mirvakili, I. W. Hunter, Adv. Mater. 2018, 30.

[50] C. S. Haines, N. Li, G. M. Spinks, A. E. Aliev, J. Di, R. H. Baughman, Proc. Natl. Acad. Sci. U S A 2016, 113, 11709.

[51] J. Foroughi, G. M. Spinks, G. G. Wallace, J. Oh, M. E. Kozlov, S. Fang, T. Mirfakhrai, J. D. Madden, M. K. Shin, S. J. Kim, R. H. Baughman, Science 2011, 334, 494.

[52] a) J. A. Lee, Y. T. Kim, G. M. Spinks, D. Suh, X. Lepro, M. D. Lima, R. H. Baughman, S. J. Kim, Nano Lett. 2014, 14, 2664; b) J. A. Lee, N. Li, C. S. Haines, K. J. Kim, X. Lepro, R. Ovalle-Robles, S. J. Kim, R. H. Baughman, Adv. Mater. 2017, 29.

[53] O. C. Compton, S. T. Nguyen, Small 2010, 6, 711.

[54] J. Qiao, J. Di, S. Zhou, K. Jin, S. Zeng, N. Li, S. Fang, Y. Song, M. Li, R. H. Baughman, Q. Li, Small 2018, 14, e1801883.

[55] K. J. Kim, J. S. Hyeon, H. Kim, T. J. Mun, C. S. Haines, N. Li, R. H. Baughman, S. J. Kim, ACS Appl. Mater. Interfaces 2019, 11, 13533.

[56] M. D. Lima, N. Li, M. Jung de Andrade, S. Fang, J. Oh, G. M. Spinks, M. E. Kozlov, C. S. Haines, D. Suh, J. Foroughi, S. J. Kim, Y. Chen, T. Ware, M. K. Shin, L. D. Machado, A. F. Fonseca, J. D. Madden, W. E. Voit, D. S. Galvao, R. H. Baughman, Science 2012, 338, 928.

[57] H. Kim, J. A. Lee, H. J. Sim, M. D. Lima, R. H. Baughman, S. J. Kim, Nanomicro Lett. 2016, 8, 254.

[58] a) S. Ghosh, D. Khastgir, A. K. Bhowmick, J. Appl. Polym. Sci. 1998, 67, 2015; b) C. D. Han, J. Kim, J. K. Kim, S. G. Chu, Macromolecules 1989, 22, 3443.

[59] C. H. Kwon, K. Y. Chun, S. H. Kim, J. H. Lee, J. H. Kim, M. D. Lima, R. H. Baughman, S. J. Kim, Nanoscale 2015, 7, 2489.

[60] Y. Song, S. Zhou, K. Jin, J. Qiao, D. Li, C. Xu, D. Hu, J. Di, M. Li, Z. Zhang, Q. Li, Nanoscale 2018, 10, 4077.

[61] a) Q. Li, M. Horn, Y. Wang, J. MacLeod, N. Motta, J. Liu, Materials (Basel) 2019, 12;

b) H. Toshiyoshi, S. Ju, H. Honma, C. H. Ji, H. Fujita, Sci. Technol. Adv. Mater. 2019, 20, 124; c) Y. Zi, J. Wang, S. Wang, S. Li, Z. Wen, H. Guo, Z. L. Wang, Nat. Commun. 2016, 7, 10987.

[62] a) M. Hamedi, R. Forchheimer, O. Inganas, Nat. Mater. 2007, 6, 357; b) L. Hu, M. Pasta, F. L. Mantia, L. Cui, S. Jeong, H. D. Deshazer, J. W. Choi, S. M. Han, Y. Cui, Nano Lett. 2010, 10, 708.

[63] K. Wang, Q. Meng, Y. Zhang, Z. Wei, M. Miao, Adv. Mater. 2013, 25, 1494.

[64] a) Z. Lou, Li, L. Wang, G. Shen, Small 2017, 13; b) X. Q. Wang, C. F. Tan, K. H. Chan, X. Lu, L. Zhu, S. W. Kim, G. W. Ho, Nat. Commun. 2018, 9, 3438.

[65] a) X. Pu, W. Hu, Z. L. Wang, Small 2018, 14; b) F. Yi, J. Wang, X. Wang, S. Niu, S. Li, Q. Liao, Y. Xu, Z. You, Y. Zhang, Z. L. Wang, ACS Nano 2016, 10, 6519.

[66] a) Q. Z. Zhang, D. Zhang, Z. C. Miao, X. L. Zhang, S. L. Chou, Small 2018, 14, e1702883; b) E. Frackowiak, Phys. Chem. Chem. Phys. 2007, 9, 1774.

[67] P. Simon, Y. Gogotsi, Nat. Mater. 2008, 7, 845.

[68] L. Wang, X. Hu, Chem. Asian J. 2018, 13, 1518.

[69] J. A. Lee, M. K. Shin, S. H. Kim, S. J. Kim, G. M. Spinks, G. G. Wallace, R. OvalleRobles, M. D. Lima, M. E. Kozlov, R. H. Baughman, ACS Nano 2012, 6, 327.

[70] F. Su, M. Miao, H. Niu, Z. Wei, ACS Appl. Mater. Interfaces 2014, 6, 2553. 
[71] a) H. Jiang, C. Li, T. Sun, J. Ma, Nanoscale 2012, 4, 807; b) X. Lu, M. Yu, G. Wang, T. Zhai, S. Xie, Y. Ling, Y. Tong, Y. Li, Adv. Mater. 2013, 25, 267; c) Z. Tang, C. H. Tang, H. Gong, Adv. Funct. Mater. 2012, 22, 1272.

[72] a) Z. B. Lei, J. T. Zhang, X. S. Zhao, J. Mater. Chem. 2012, 22, 153; b) X. Zhang, D. D. Zhao, Y. Q. Zhao, P. Y. Tang, Y. L. Shen, C. L. Xu, H. L. Li, Y. Xiao, J. Mater. Chem. A 2013, 1, 3706.

[73] a) H. Gao, F. Xiao, C. B. Ching, H. Duan, ACS Appl. Mater. Interfaces 2012, 4, 2801; b) A. Sumboja, C. Y. Foo, X. Wang, P. S. Lee, Adv. Mater. 2013, 25, 2809; c) Z. Lu, Y. Chao, Y. Ge, J. Foroughi, Y. Zhao, C. Wang, H. Long, G. G. Wallace, Nanoscale 2017, 9, 5063.

[74] C. Choi, J. A. Lee, A. Y. Choi, Y. T. Kim, X. Lepro, M. D. Lima, R. H. Baughman, S. J. Kim, Adv. Mater. 2014, 26, 2059.

[75] J. H. Kim, C. Choi, J. M. Lee, M. J. de Andrade, R. H. Baughman, S. J. Kim, Sci. Rep. 2018, 8, 13309.

[76] F. Su, M. Miao, Nanotechnology 2014, 25, 135401.

[77] F. Su, X. Lv, M. Miao, Small 2015, 11, 854.

[78] W. Wei, X. Cui, W. Chen, D. G. Ivey, Chem. Soc. Rev. 2011, 40, 1697.

[79] a) M. Okubo, A. Sugahara, S. Kajiyama, A. Yamada, Acc. Chem. Res. 2018, 51, 591; b) C. J. Zhang, L. McKeon, M. P. Kremer, S. H. Park, O. Ronan, A. Seral-Ascaso, S. Barwich, C. O. Coileain, N. McEvoy, H. C. Nerl, B. Anasori, J. N. Coleman, Y. Gogotsi, V. Nicolosi, Nat. Commun. 2019, 10, 1795.

[80] X. Li, C. Wang, Y. Cao, G. Wang, Chem. Asian J. 2018, 13, 2742.

[81] M. R. Lukatskaya, O. Mashtalir, C. E. Ren, Y. Dall'Agnese, P. Rozier, P. L. Taberna, M. Naguib, P. Simon, M. W. Barsoum, Y. Gogotsi, Science 2013, 341, 1502.

[82] a) J. Zhang, S. Seyedin, Z. Gu, W. Yang, X. Wang, J. M. Razal, Nanoscale 2017, 9, 18604; b) S. Seyedin, E. R. S. Yanza, J. M. Razal, J. Mater. Chem. A 2017, 5, 24076.

[83] Z. Wang, S. Qin, S. Seyedin, J. Zhang, J. Wang, A. Levitt, N. Li, C. Haines, R. OvalleRobles, W. Lei, Y. Gogotsi, R. H. Baughman, J. M. Razal, Small 2018, 14, e1802225.

[84] J. A. Lee, M. K. Shin, S. H. Kim, H. U. Cho, G. M. Spinks, G. G. Wallace, M. D. Lima, X. Lepro, M. E. Kozlov, R. H. Baughman, S. J. Kim, Nat. Commun. 2013, 4, 1970.

[85] D. Zhang, M. Miao, H. Niu, Z. Wei, ACS Nano 2014, 8, 4571.

[86] D. Zhang, Y. Wu, T. Li, Y. Huang, A. Zhang, M. Miao, ACS Appl. Mater. Interfaces 2015, 7, 25835.

[87] K. M. Kim, J. A. Lee, H. J. Sim, K. A. Kim, R. Jalili, G. M. Spinks, S. J. Kim, Nanoscale 2016, 8, 1910.

[88] L. Kou, T. Huang, B. Zheng, Y. Han, X. Zhao, K. Gopalsamy, H. Sun, C. Gao, Nat. Commun. 2014, 5, 3754.

[89] T. Sekitani, Y. Noguchi, K. Hata, T. Fukushima, T. Aida, T. Someya, Science 2008, 321, 1468.

[90] M. K. Shin, J. Oh, M. Lima, M. E. Kozlov, S. J. Kim, R. H. Baughman, Adv. Mater. 2010, 22, 2663.

[91] W. Son, S. Chun, J. M. Lee, Y. Lee, J. Park, D. Suh, D. W. Lee, H. Jung, Y. J. Kim, Y. Kim, S. M. Jeong, S. K. Lim, C. Choi, Nat. Commun. 2019, 10, 426.

[92] C. Choi, S. H. Kim, H. J. Sim, J. A. Lee, A. Y. Choi, Y. T. Kim, X. Lepro, G. M. Spinks, R. H. Baughman, S. J. Kim, Sci. Rep. 2015, 5, 9387.

[93] C. Choi, K. M. Kim, K. J. Kim, X. Lepro, G. M. Spinks, R. H. Baughman, S. J. Kim, Nat. Commun. 2016, 7, 13811.

[94] C. Choi, J. M. Lee, S. H. Kim, S. J. Kim, J. Di, R. H. Baughman, Nano Lett. 2016, 16, 7677. 
[95] a) Z. Chai, N. Zhang, P. Sun, Y. Huang, C. Zhao, H. J. Fan, X. Fan, W. Mai, ACS Nano 2016, 10, 9201; b) R. A. Kishore, S. Priya, Materials (Basel) 2018, 11; c) P. Talemi, M. Delaigue, P. Murphy, M. Fabretto, ACS Appl. Mater. Interfaces 2015, 7, 8465.

[96] a) C. A. Hewitt, A. B. Kaiser, S. Roth, M. Craps, R. Czerw, D. L. Carroll, Nano Lett. 2012, 12, 1307; b) Q. Yao, L. Chen, W. Zhang, S. Liufu, X. Chen, ACS Nano 2010, 4, 2445; c) C. Yu, Y. S. Kim, D. Kim, J. C. Grunlan, Nano Lett. 2008, 8, 4428; d) D. Kim, Y. Kim, K. Choi, J. C. Grunlan, C. H. Yu, Acs Nano 2010, 4, 513; e) R. Haggenmueller, C. Guthy, J. R. Lukes, J. E. Fischer, K. I. Winey, Macromolecules 2007, 40, 2417.

[97] J. A. Lee, A. E. Aliev, J. S. Bykova, M. J. de Andrade, D. Kim, H. J. Sim, X. Lepro, A. A. Zakhidov, J. B. Lee, G. M. Spinks, S. Roth, S. J. Kim, R. H. Baughman, Adv. Mater. 2016, 28, 5038.

[98] J. Choi, Y. Jung, S. J. Yang, J. Y. Oh, J. Oh, K. Jo, J. G. Son, S. E. Moon, C. R. Park, H. Kim, ACS Nano 2017, 11, 7608.

[99] a) W. M. Zhang, A. I. Minett, M. Gao, J. Zhao, J. M. Razal, G. G. Wallace, T. Romeo, J. Chen, Adv. Energy Mater. 2011, 1, 671; b) Z. H. Wen, Q. Wang, J. H. Li, Adv. Funct. Mater. 2008, 18, 959; c) Z. Q. Tian, S. H. Lim, C. K. Poh, Z. Tang, Z. T. Xia, Z. Q. Luo, P. K. Shen, D. Chua, Y. P. Feng, Z. X. Shen, J. Y. Lin, Adv. Energy Mater. 2011, $1,1205$.

[100] a) S. C. Hou, X. Cai, Y. P. Fu, Z. B. Lv, D. Wang, H. W. Wu, C. Zhang, Z. Z. Chu, D. C. Zou, J. Mater. Chem. 2011, 21, 13776; b) T. Chen, L. B. Qiu, Z. B. Cai, F. Gong, Z. B. Yang, Z. S. Wang, H. S. Peng, Nano Lett. 2012, 12, 2568.

[101] S. Zhang, C. Y. Ji, Z. Q. Bian, P. R. Yu, L. H. Zhang, D. Y. Liu, E. Z. Shi, Y. Y. Shang, H. T. Peng, Q. Cheng, D. Wang, C. H. Huang, A. Y. Cao, Acs Nano 2012, 6, 7191.

[102] D. Y. Liu, M. Y. Zhao, Y. Li, Z. Q. Bian, L. H. Zhang, Y. Y. Shang, X. Y. Xia, S. Zhang, D. Q. Yun, Z. W. Liu, A. Y. Cao, C. H. Huang, Acs Nano 2012, 6, 11027.

[103] X. H. Yu, J. Pan, J. Deng, J. Zhou, X. M. Sun, H. S. Peng, Adv. Mater. 2016, 28, 10744.

[104] a) Q. Zheng, B. Shi, Z. Li, Z. L. Wang, Adv. Sci. (Weinh) 2017, 4, 1700029; b) G. T. Hwang, M. Byun, C. K. Jeong, K. J. Lee, Adv. Healthc. Mater. 2015, 4, 646; c) R. Hinchet, W. Seung, S. W. Kim, ChemSusChem 2015, 8, 2327.

[105] S. H. Kim, C. S. Haines, N. Li, K. J. Kim, T. J. Mun, C. Choi, J. T. Di, Y. J. Oh, J. P. Oviedo, J. Bykova, S. L. Fang, N. Jiang, Z. F. Liu, R. Wang, P. Kumar, R. Qiao, S. Priya, K. Cho, M. Kim, M. S. Lucas, L. F. Drummy, B. Maruyama, D. Y. Lee, X. Lepro, E. L. Gao, D. Albarq, R. Ovalle-Robles, S. J. Kim, R. H. Baughman, Science 2017, 357, 773.

[106] B. Gorissen, D. Reynaerts, S. Konishi, K. Yoshida, J. W. Kim, M. De Volder, Adv. Mater. 2017, 29.

[107] a) G. Alici, Expert Rev. Med. Devices 2015, 12, 703; b) A. W. Feinberg, Annu. Rev. Biomed. Eng. 2015, 17, 243.

[108] C. Laschi, M. Cianchetti, Front. Bioeng. Biotechnol. 2014, 2, 3. 


\section{WILEY-VCH}

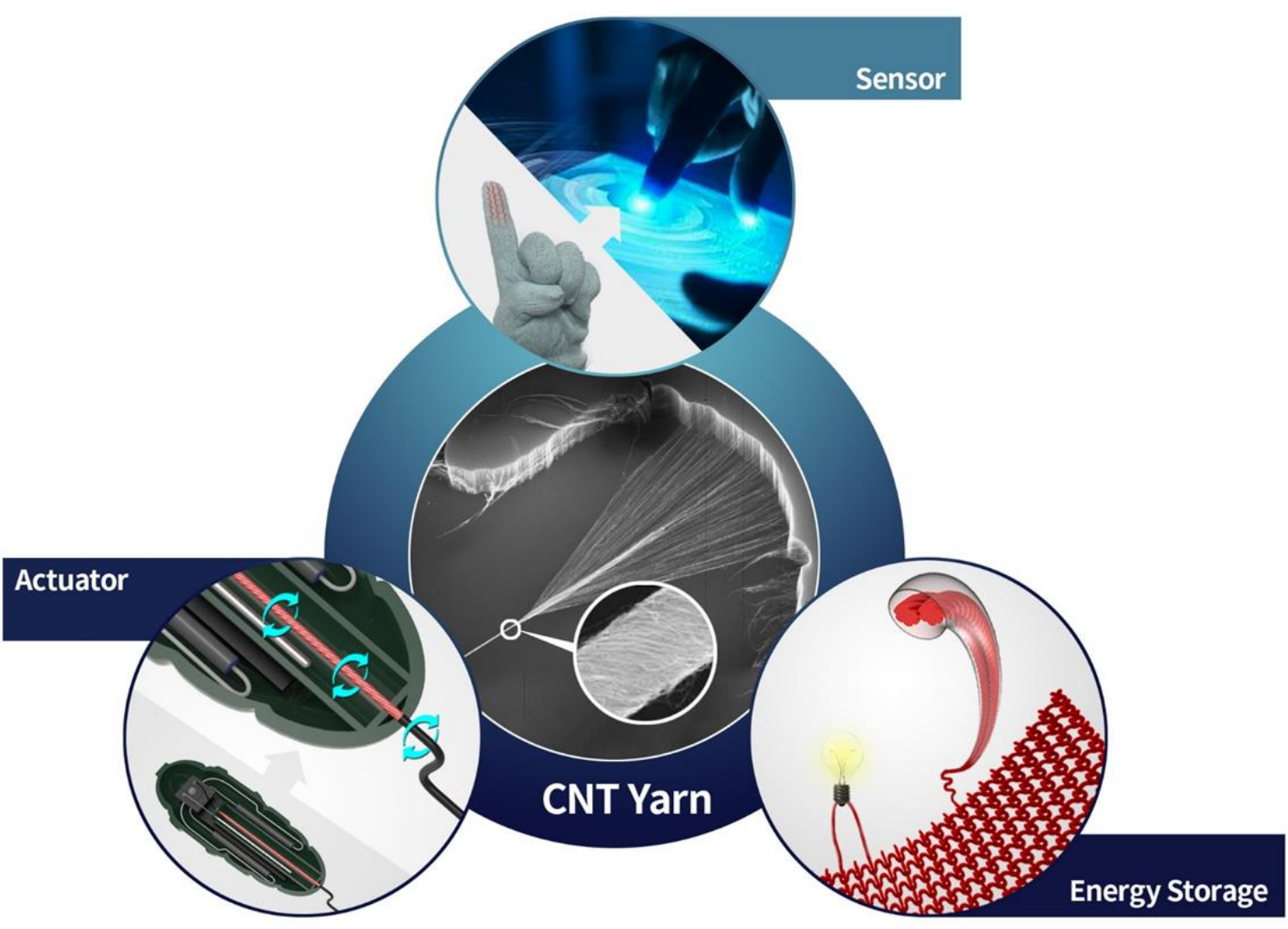

Figure 1. Schematic illustration of various functions of CNT yarns as a sensor, actuator, and energy storage. 


\section{WILEY-VCH}
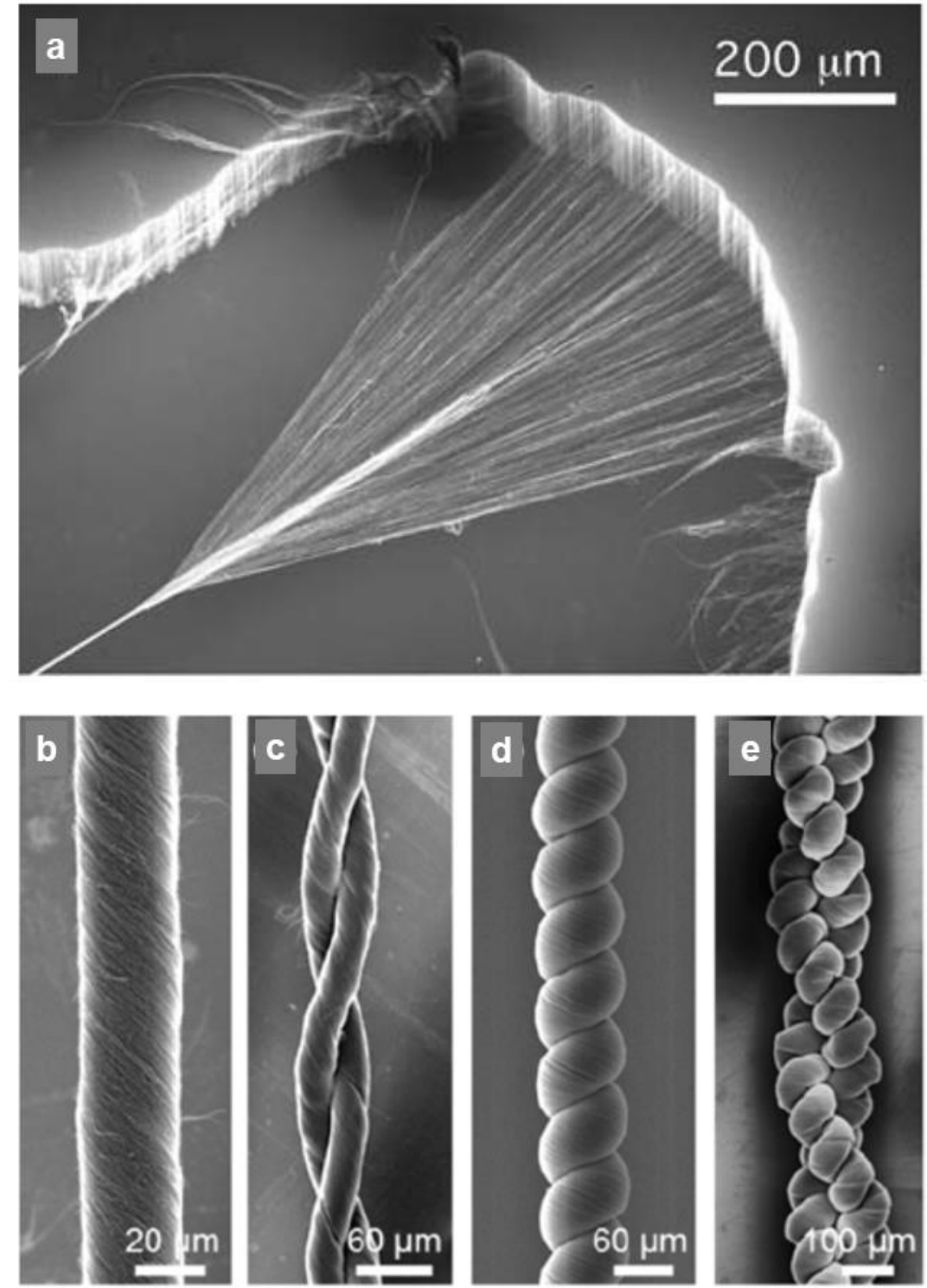

Figure 2. CNT forest and CNT yarn structures. a) SEM image of CNT yarn in the spinning process from CNT forest. Reproduced with permission. ${ }^{[9]}$ Copyright 2004, The American Association for the Advancement of Science. b-e) SEM images of single CNT yarn (b), two-ply CNT yarns (c), coiled CNT yarns (d), two-ply coiled yarns (e). Reproduced with permission. ${ }^{[59 a]}$ Copyright 2014, American Chemical Society. 


\section{WILEY-VCH}

a

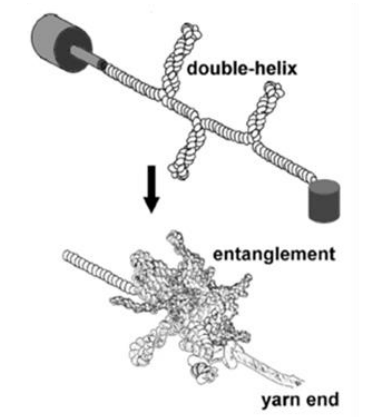

b

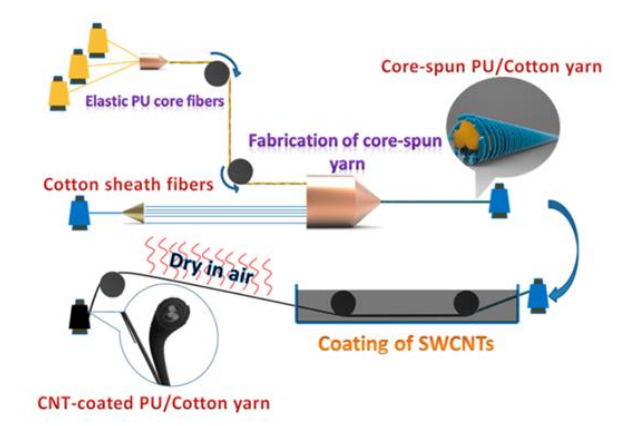

C

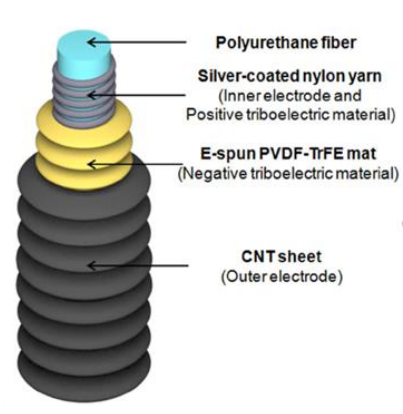

d

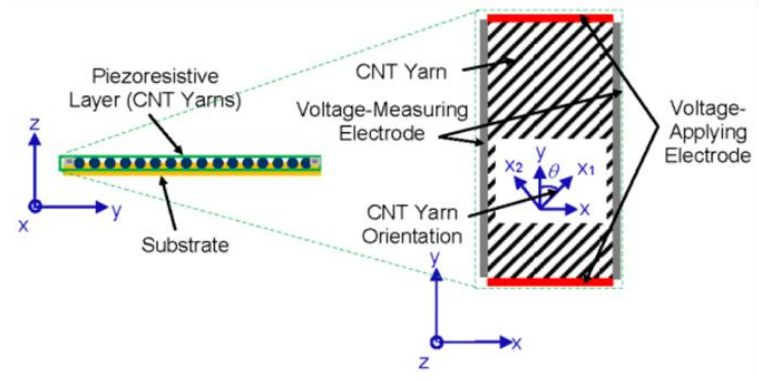

e

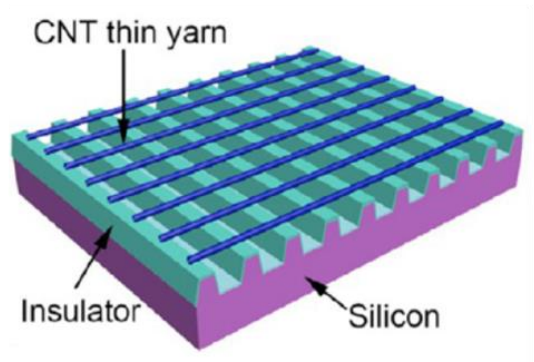




\section{WILEY-VCH}

Figure 3. Various fabrications of CNT-based fibers and CNT yarns as a stretchable strain

sensor. a) Schematic illustration of the spinning process of entangled CNT strain sensor. Reproduced with permission. ${ }^{[26]}$ Copyright 2013, American Chemical Society. b) Schematic illustration of the fabrication process of the PU/cotton/CNT yarn strain sensor. Reproduced with permission. ${ }^{[27]}$ Copyright 2016, American Chemical Society. c) Schematic illustration of CNT-based triboelectric fiber strain sensor. Reproduced with permission. ${ }^{[28]}$ Copyright 2016, Springer Nature. d) Schematic illustration of cross-section of the strain sensor comprising CNT yarns. Reproduced with permission. ${ }^{[29]}$ Copyright 2018, MDPI. e) Schematic illustration of CNT thermoacoustic strain sensor. Reproduced with permission. ${ }^{[30]}$ Copyright 2013, American Chemical Society.
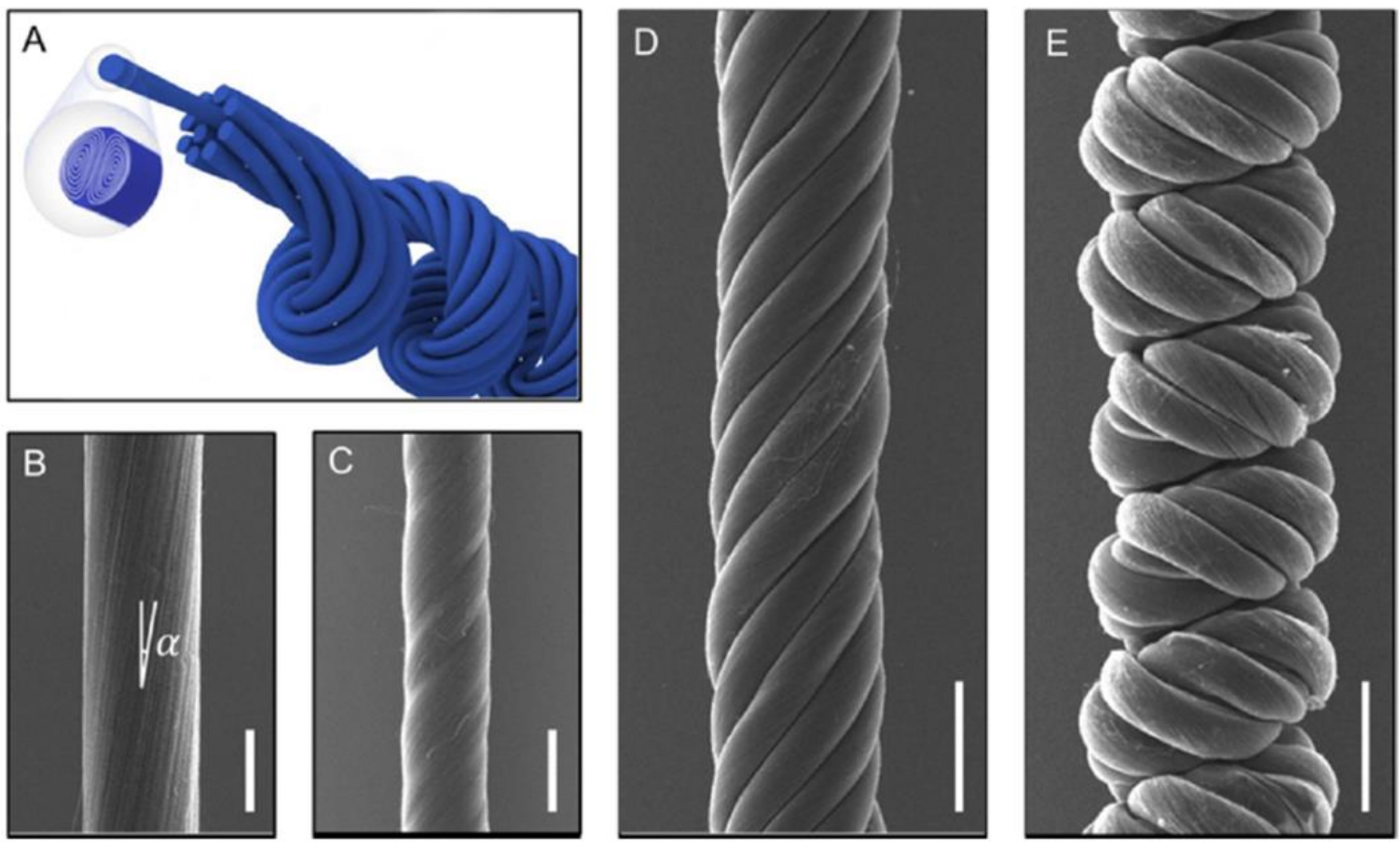


\section{WILEY-VCH}

Figure 4. CNT yarn muscle configuration and morphology. a) Schematic illustration of the hierarchical structure of a coiling plies of twist-released CNT yarns. b) SEM image of a twisted CNT yarn having a bias angle of $9.4^{\circ}$. c) SEM image of a fully twisted precursor yarn having a bias angle of $44.9^{\circ}$. d) SEM image of a twisted multiply CNT yarn. e) SEM image of a coiled multiply of twist-released CNT yarns. Scale bars indicate $50 \mu \mathrm{m}(\mathrm{b}$ and c) and $100 \mu \mathrm{m}$ (d and e). Reproduced with permission. ${ }^{[55]}$ Copyright 2019, American Chemical Society. 


\section{WILEY-VCH}

a

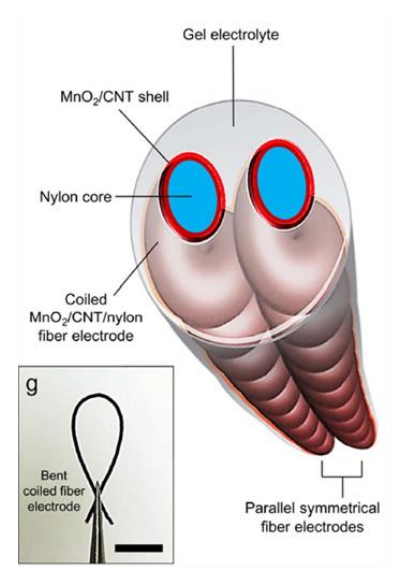

b

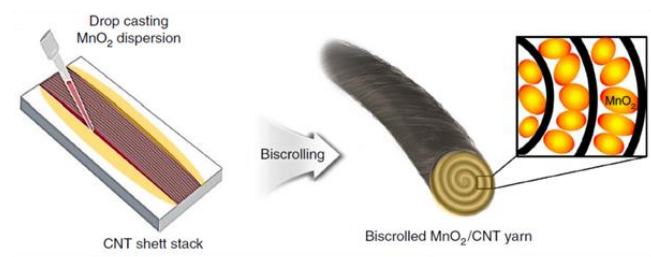

CNT shett stack

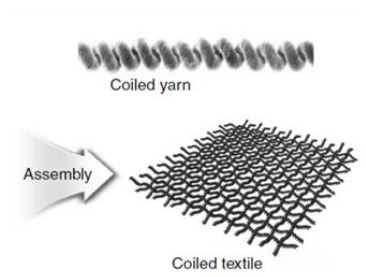

C

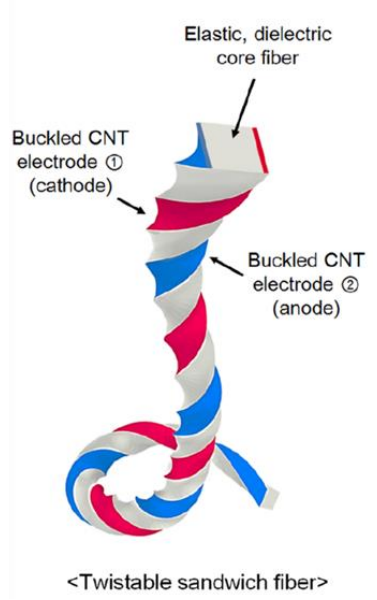

Figure 5. Various fabrications of CNT-based fiber and CNT yarns as a stretchable supercapacitor. a) Schematic illustration of Schematic illustration for the supercapacitor, which consist of two symmetric coiled $\mathrm{MnO}_{2} / \mathrm{CNT} /$ nylon fiber electrodes in the gel electrolyte. Reproduced with permission. ${ }^{[92]}$ Copyright 2015, Springer Nature. b) Schematic illustration of the fabrication of a biscrolled $\mathrm{MnO}_{2} / \mathrm{CNT}$ yarn electrode, and coiled and woven supercapacitor by a biscrolled yarn. Reproduced with permission. ${ }^{[93]}$ Copyright 2016, Springer Nature. c) Schematic illustration of a twist-induced rectangular fiber, which consist of a rubber core and two symmetric CNT electrodes. Reproduced with permission. ${ }^{[94]}$ Copyright 2016, American Chemical Society. 


\section{WILEY-VCH}

a

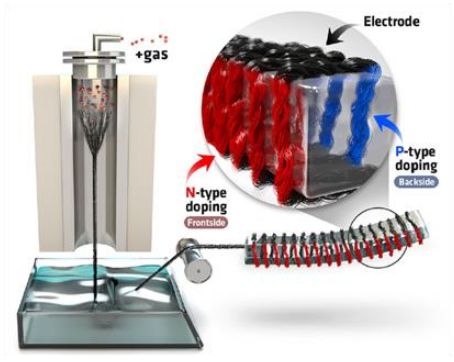

Thermoelectric conversion b

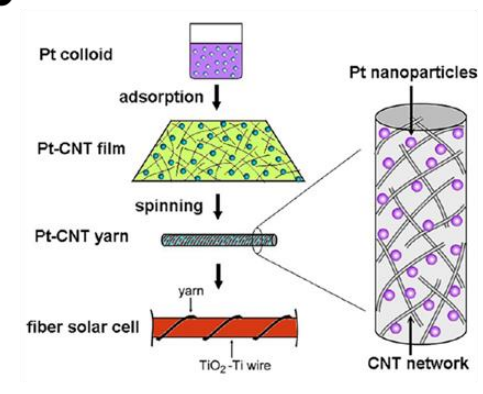

Photoelectric conversion
C

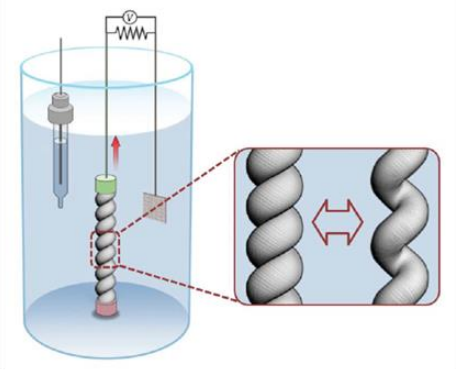

Mechanoelectric conversion 


\section{WILEY-VCH}

Figure 6. Various fabrications of CNT yarns as energy harversters. a) Schematic illustration of the flexible thermoelectric generator based on CNT yarn. Reproduced with permission. ${ }^{[98]}$ Copyright 2017, American Chemical Society. b) Schematic illustration of the fabrication process of Pt-CNT yarn on $\mathrm{TiO}_{2}$ - $\mathrm{Ti}$ wire for photoelectric conversion. Reproduced with permission. ${ }^{[101]}$ Copyright 2012, American Chemical Society. c) Schematic illustration of a coiled CNT yarn harvester electrode and counter and reference electrodes in an electrochemical bath. Reproduced with permission. ${ }^{[105]}$ Copyright 2017, American Chemical Society. 